Address for Correspondence: Stefano Benedini, Endocrinology Unit, IRCCSPoliclinico San Donato, Via Morandi 30, 20097, San Donato Milanese, Milan Italy.

E-mail: stefano.benedini@unimi.it

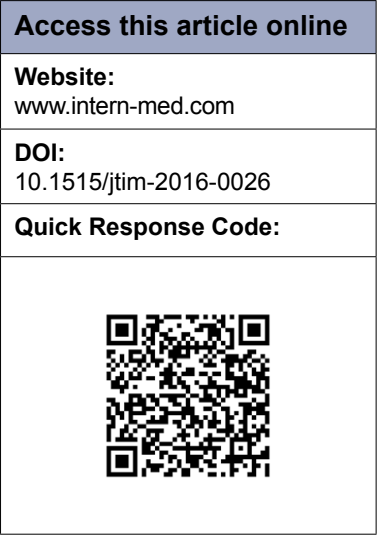

\title{
Lipodystrophy HIV-related and FGF21: A new marker to follow the progression of lipodystrophy?
}

\author{
Stefano Benedini ${ }^{1,2}$, Livio Luzi ${ }^{1,2,3}$ \\ 'Department of Biomedical Sciences and Health, Università degli Studi di Milano, Milan, Italy; \\ ${ }^{2}$ Endocrinology Unit, IRCCS Policlinico San Donato, San Donato M.se (MI), Italy; \\ ${ }^{3}$ Metabolism Research Center, IRCCS Policlinico San Donato, Milan, Italy
}

\section{ABSTRACT}

Recently new evidence about fibroblast growth factor 21 (FGF21) highlights the opportunities to use this molecule in new pharmaceutical formulations to combat type 2 diabetes and metabolic syndrome. It is well known that HIV is per se a condition of insulin resistance and in particular the patient with HIV-related lipodystrophy has a condition strictly related to metabolic syndrome. Lipodystrophy is associated with severe metabolic side effects, including dyslipidemia, hepatic insulin resistance, and lipid oxidation impairment. Research carried out showed that FGF21 levels were significantly increased in untreated HIV-1-infected patients and the increase was much marked in HIV-1-infected antiretroviral-treated patients that have developed lipodystrophy and in the patients with greatest metabolic alterations. FGF21 is expressed mainly by the liver, but also by other tissues such as the thymus, adipose tissue, and skeletal muscle. Therefore, many researchers have considered the investigation of possible variations of FGF21 in patients with significant alterations in body composition both in regard to fat mass and lean mass. In the light of the possible interactions between FGF21 and metabolic syndrome, it seems interesting to evaluate the implication of this hormone in patients with HIV-related lipodystrophy who have a severe metabolic picture of insulin resistance with important alterations in body composition.

Key words: insulin sensitivity, adipose tissue, lipid oxidation, hypothalamus

\section{INTRODUCTION}

Human immunodeficiency virus (HIV) lipodystrophy is characterized by changes in fat distribution and increase in insulin resistance. A disturbance of lipid metabolism, as the consequence of viral infection, or the association of virus-antiretroviral genetic therapy background, seems to play a central role in the pathogenesis of this syndrome. Also insulin resistance is one of the earliest metabolic alterations present in patients with HIV and with protease inhibitors (PIs) therapy ${ }^{[1]}$. In the last two decades, PIs therapy improved survival and quality of life of HIV-infected patients. These patients often develop a syndrome characterized by peripheral lipoatrophy, trunk fat accumulation, and metabolic alterations. The causes of this clinical picture are still not completely defined. The lipodystrophy in HIV-1 patients in antiretroviral treatment is associated with peripheral fat wasting and central adiposity, dyslipidemia, and insulin resistance but also with increase intramuscular fat accumulation, related to development of the insulin resistance syndrome ${ }^{[2]}$.

The distribution of fat in humans is governed by many factors, including genetics, hormonal pathway in particular sex hormones, age, environmental factors (such as diet, exercise, integrators and drugs), and associated diseases.

FGF21 is a hormone able to determine some metabolic adaptations essential for 
the homeostasis of our body. In particular, the ability to increase lipid oxidation from the liver, the stimulus to gluconeogenesis and ketogenesis appear as the fundamental mechanisms during the fasting period ${ }^{[3]}$.

FGF21 is also able to bind to receptors in the hypothalamus, producing an increase in energy expenditure and improvement of insulin sensitivity. In humans, it appears that the transport of FGF21 in the central nervous system is mediated by transporters given the size of this protein ${ }^{[4]}$. A critical metabolic feature of FGF21 is its capacity to sensitize insulin action in vivo ${ }^{[5]}$.

As metabolic modulator, FGF21 seems to play an important role in lipolysis during starvation, in fatty acid oxidation and in promoting ketogenesis ${ }^{[6,7]}$. FGF21 seems to be able to increase the insulin receptors in the liver, resulting in an improvement of insulin sensitivity in toto and, in adipose tissue, appears to inhibit the lipolysis in adipocytes with consequent reduction of circulating fatty acids. These two mechanisms cause an improvement in insulin sensitivity in humans. ${ }^{[8,9]}$

\section{HIV AND LIPODYSTROPHY}

In HIV patients on antiretroviral therapy with protease inhibitors, alterations in glucose and lipid metabolism are well known ${ }^{[1]}$. Along with the metabolic alterations morphological changes often accompany these individuals in particularly alteration the redistribution of the fat tissue.

In particular, in this syndrome, there is a severe lipoatrophy ${ }^{[10]}$. Lipoatrophy is primarily subcutaneous fat loss. Fat deposition in patients with HIV occurs in the visceral depot (intra-abdominally), breasts, and dorsocervical area of the neck. Sometimes, some patients have fat deposits in the form of lipomas. The term "HIVassociated adipose redistribution syndrome" has been used to define a distinct subset of general lipodystrophy, which is characterized by the abnormal accumulation of visceral adipose tissue, with or without comorbid lipoatrophy and metabolic abnormalities such as alteration of lipid profile or insulin resistance ${ }^{[11]}$.

In fact, the term "lipodystrophy syndrome" in association with HIV was introduced to describe a complex medical picture, including an apparent abnormal fat redistribution and metabolic alterations present in HIV patients receiving protease inhibitor therapy.

The adipose tissue in HIV patients with lipodystrophy is reduced in some locations with a buildup in other, and there is a reduction of the tissue under the skin on the face and limbs with a simultaneous increase in visceral fat ${ }^{[12]}$. In patients with HIV lipodystrophy, there are alterations in body composition that remind, in part, those in patients with Cushing's syndrome in which there is an increase in visceral fat and a decrease of subcutaneous fat. Although there is not a situation of real hypercorticism in HIV patients ${ }^{[13]}$, hypertrophic changes consist of excess fat deposition, common around the abdominal region, but also in the neck, in the supraclavicular fossae, or in the subcutaneous tissues of the posterior neck, between the scapulae. Atrophic changes consist of subcutaneous fat atrophy in the legs, buttocks, arms, and nasolabial and malar fat pads. The cosmetically disfiguring changes that occur with lipodystrophy may have a significant impact on quality of life and relational activities ${ }^{[14]}$. Dyslipidemia was reported

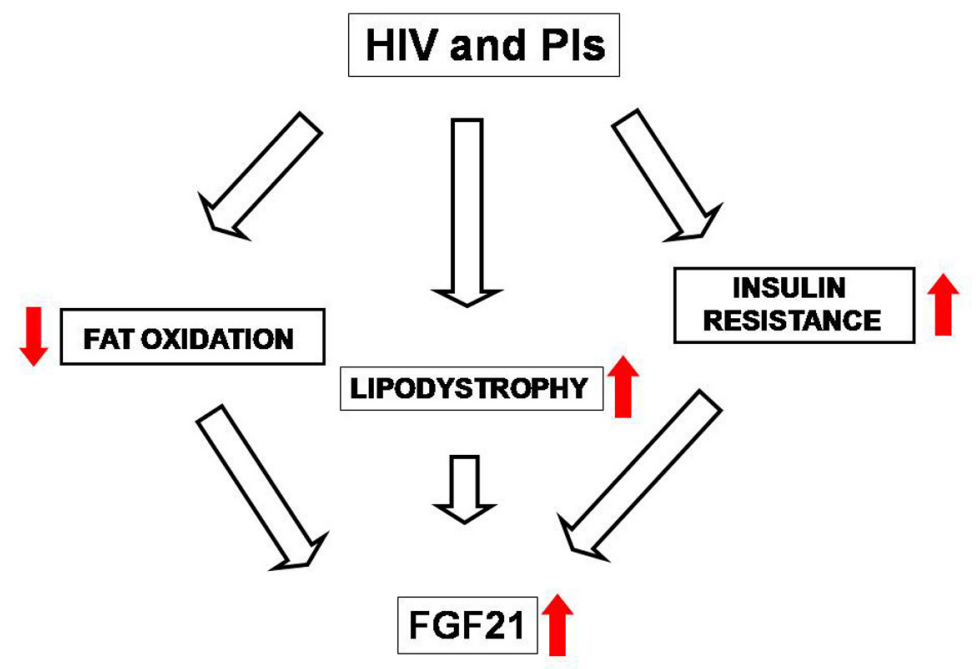

Figure 1: Morphological and metabolic alterations in patients with HIV-related lipodystrophy. The increase of insulin resistance, decrease of lipid oxidation, and redistribution of fat tissue has the capacity to increase the values of plasma FGF21. Therefore, this protein could be a good marker for the development and monitoring of lipodystrophy in HIV-infected patients. HIV: human immunodeficiency virus; FGF21: fibroblast growth factor 21; Pls: protease inhibitors. 
in HIV-infected individuals regardless of drug therapy ${ }^{[15]}$. PIs therapy of HIV-infected individuals was associated with increased total cholesterol, non-HDL-C, triglyceride, IDL, and VLDL cholesterol. The increased VLDL levels were due to increased concentrations of large VLDL particles, which include chylomicron remnants. Furthermore, the individuals receiving PIs therapy were shown to have altered parameters of endothelial dysfunction ${ }^{[16]}$.

Today, unfortunately, there are very few effective treatments to subvert the picture of lipodystrophy in more severe forms that affect patients with HIV. Several forms of plastic surgery have been adopted in patients with faciallipoatrophy ${ }^{[17]}$. Other therapies, including implants, have been used anecdotally in other body sites ${ }^{[18]}$.

\section{FGF2 1, METABOLISM, AND LIPODYSTROPHY}

FGF21 is classified as fibroblast growth factor because it is very similar structurally to this category of proteins but, in vivo, seems to have no ability to stimulate cell growth. FGF21 seems to act primarily on adipose tissue, liver, and pancreas. This protein has endocrine actions on different target organs producing important metabolic changes ${ }^{[7]}$.

In particular, it plays a key role in activating mechanisms of homeostasis during fasting to keep glucose levels within the normal range ${ }^{[5]}$. Therefore it increases gluconeogenesis, ketogenesis, and fatty acid oxidation ${ }^{[6}$. Also it seems to be involved in the control of blood glucose by increasing insulin sensitivity ${ }^{[3,5]}$.
FGF21 is a powerful catabolic protein that is capable of potently inducing energy expenditure, thus leading to a negative whole-body energy balance ${ }^{[19]}$. It seems clear today that FGF21 acts in synergy with other hormones with metabolic action as insulin, glucagon, leptin, glucagon-like peptide, adiponectin, growth hormone, glucagon-like peptide 1 (GLP1), and insulin-like growth factor 1 (IGF1) and then it seems logical to think of a potential therapeutic role in the metabolic syndrome or in overt diabetes ${ }^{[20]}$.

In an interesting study performed on the animal model, the authors demonstrates that both liver mRNA expression and serum level of "anti-diabetic factor" FGF21 are markedly up-regulated in lipodystrophic A-ZIP F-1 mice ${ }^{[21]}$.

Unfortunately, most of the studies on the action of FGF21 were performed in vitro or in animal models, particularly in murine and nonhuman primate models.

Only two FGF21 analog are study in humans ${ }^{[22,23]}$ as been performed and the results of these studies shown a decrease of body weight, decrease of insulin resistance, decrease of triglycerides, and LDL cholesterol with increase of HDL.

FGF21 can act on brown adipose tissue (BAT) as an autocrine, paracrine, and endocrine agent, activating BAT thermogenesis ${ }^{[24]}$.

In the past, various measures have been proposed in both pharmacology and lifestyle to improve the metabolic and body fat distribution in patients with lipodystrophy.

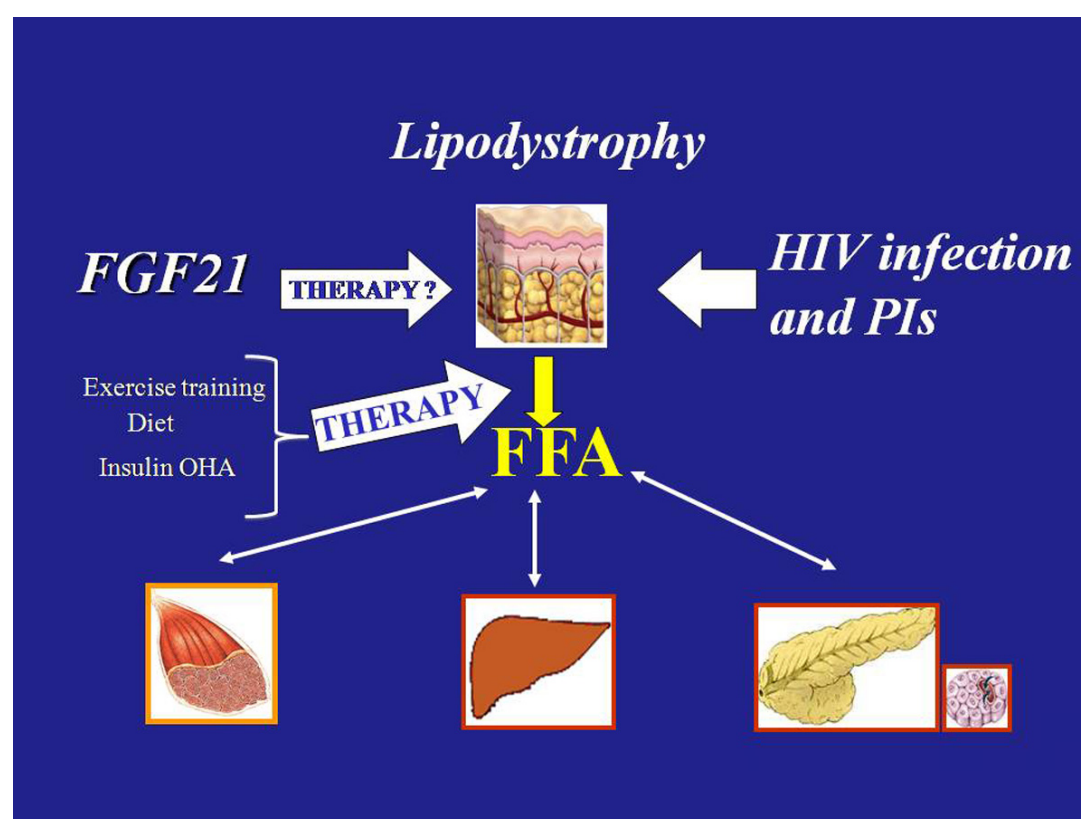

Figure 2: In patients with HIV lipodystrophy alterations of lipid metabolism appear as central in the development of this syndrome, in particular the increase of circulating fatty acids causes a deposition of lipids in the liver, intramuscular and in the island of Langerhans with a consequent increase of insulin resistance. This process could be countered by the use of FGF21 analogs. HIV: human immunodeficiency virus; FGF21: fibroblast growth factor 21; Pls: protease inhibitors; FFA: free fatty acid. 
In particular, the modification of lifestyle (3 h of physical activity at moderate intensity per-week) was studied in patients with HIV-related lipodystrophy with significant improvement of metabolic picture after six months of intervention, and, in this study, it was concluded that intensive lifestyle modification improved important cardiovascular risk indices in HIV patients with the metabolic syndrome ${ }^{[25]}$.

In another study performed by our group, it has been highlighted that in patients with lipodystrophy in acquired immune deficiency syndrome, eight months of 1-acetylcarnitine treatment increased lipid oxidation, decreased intramyocellular triglyceride content, and induced a more physiological distribution of fat tissue ${ }^{[26]}$. In 2010, a careful meta-analysis of the effects of metformin in this patient population revealed favorable impacted outcomes consistent in significant reductions in fasting insulin, fasting triglycerides, body mass index, and waistto-hip ratio ${ }^{[27]}$. In a recent paper, it has been demonstrated that FGF-21 mRNA expression is increased in skeletal muscle in patients with HIV-related lipodystrophy and that FGF-21 mRNA expression in muscle is associated with decreased limb fat mass, increased waist-to-hip ratio, and increased triglycerides ${ }^{[28]}$.

Another well-conducted study found highly significant correlation between FGF21 levels and indicators of altered fat distribution in lipodystrophy and a positive relationship between FGF21 levels and the extent of lipodystrophy ${ }^{[29]}$. On the contrary, there is no strict correlation between FGF21 levels and fat mass in obese patients. In particular, the association of serum FGF21 levels with BMI and fat percentage is weaker than other adipokines predominantly produced from adipose tissue, such as leptin ${ }^{[30]}$.

\section{POSSIBLE ROLE OF FGF21 IN ASSESSING THE DEGREE OF LIPODYSTROPHY}

The role of adipocytokines and of other hormones in the pathogenesis of HIV-associated lipodystrophy is still not enough understood. In particular, in our review, we focused attention on FGF21, which is expressed mainly by the liver, but also by other tissues such as adipose tissue ${ }^{[31]}$. Moreover, adipose tissue showed a large high expression of FGF receptor $1^{[32]}$ and emerged as a strong candidate to represent a predominant FGF21 target tissue. This new evidence about FGF21 highlights the opportunities to target this molecule for new pharmaceutical formulations to combat type 2 diabetes and metabolic syndrome ${ }^{[20]}$ and that a rise in circulating FGF21 has been proposed as a predictor of the development of the metabolic syndrome and type 2 diabetes mellitus ${ }^{[33]}$. We decided to propose this hormone as a marker of metabolic and morphological alterations in the patient with HIV-related lipodystrophy in order to monitor the efficacy of the proposed interventions (drugs, modification of lifestyle, and different supplements).

The study performed by Domingo and coworkers shows that the indices of fat distribution (waist-to-hip ratio and trunk/apendicular fat ratio) are closely correlated with the values of FGF21 in subjects with HIV-related lipodystropy. In the same study, it is emphasized that the positive correlation between FGF21 and HOMA-IR, triglycerides, and LDL cholesterol. It seems clear that the value of FGF21 in this group of patients could be employed on a large scale in order to assess the starting point of start of this complication and then monitoring the effectiveness of intervention proposed through dosing FGF21 over time. The opportunity to use the same assay to monitor the metabolic and morphologic distribution of fat linked to lipodystrophy looks very interesting and promising.

More studies are needed to confirm this trend highlighted by the study of Domingo ${ }^{[29]}$ but the presuppositions seem encouraging.

\section{CONCLUSION}

The hormone FGF21 seems to have a fundamental role in the modulation of the metabolism in humans also is largely produced by adipose tissue and it performed various actions directly on adipose tissue ${ }^{[34]}$. Given the severe metabolic and morphological alterations of adipose tissue in HIV-related lipodystrophy, FGF21 appears be an ideal candidate to assess the susceptibility of a patient with HIV to develop lipodystrophy. At the same time, this protein can be a promising marker for assessing the effectiveness of pharmacological and other interventions on lipodystrophy. At present, to confirm this hypothesis, it will require prospective studies on a large number of patients in order to determine the real role of FGF21 as a marker of lipodystrophy in HIV patients. The big pharmacological efforts to get the identification of a drug with similar action of FGF21could open new perspectives in the treatment of HIV-related lipodystrophy.

\section{Conflicts of Interest}

The author states that there is no conflict of interest.

\section{REFERENCES}

1. Capiluppi B, Ciuffreda D, Sciandra M, Marroni M, Tambussi G, and Lazzarin A. Metabolic disorders in a cohort of patients treated with highly aggressive antiretroviral therapies during primary HIV-1 infection. AIDS 
2000; 14:1861-2.

2. Luzi L, Perseghin G, Tambussi G, Meneghini E, Scifo P, Pagliato E, et al, LazzarinA. Intramyocellular lipid accumulation and reduced whole body lipid oxidation in HIV lipodystrophy. Am J Physiol Endocrinol Metab 2003;284:E274-80.

3. Long YC, Kharitonenkov A. Hormone-like fibroblast growth factors and metabolic regulation. Biochim Biophys Acta 2011;1812:791-5.

4. Kharitonenkov A, Larsen P. FGF21 reloaded: challenges of a rapidly growing field. Trends Endocrinol Metab 2011;22:81-6.

5. Xu J, Lloyd DJ, Hale C, Stanislaus S, Chen M, Sivits G, et al, Fibroblast growth factor 21 reverses hepatic steatosis, increases energy expenditure, and improves insulin sensitivity in diet-induced obese mice, Diabetes 2009; 58:250-9.

6. Badman MK, Pissios P, Kennedy AR, Koukos G, Flier JS, Maratos-Flier E. Hepatic fibroblast growth factor 21 is regulated by PPAR alpha and is a key mediator of hepatic lipid metabolism in ketotic states. Cell Metab 2007;5:426-37.

7. Inagaki T, Dutchak P, Zhao G, Ding X, Gautron L, Parameswara V, et al. Endocrine regulation of the fasting response by PPARalpha-mediated induction of fibroblast growth factor 21. Cell Metab 2007;5:415-25.

8. Arner P1, Pettersson A, Mitchell PJ, Dunbar JD, Kharitonenkov A, Rydén M. FGF21 attenuates lipolysis in human adipocytes- a possible link to improved insulin sensitivity. FEBS Lett 2008;582:1725-30.

9. Li X, Ge H, Weiszmann J, Hecht R, Li YS, Véniant MM, et al. Inhibition of lipolysis may contribute to the acuteregulation of plasma FFA and glucose by FGF21 in ob/ob mice. FEBS Lett 2009; 583:3230-4.

10. Finkelstein JL, Gala P, Rochford R, Glesby MJ, Mehta S. HIV/AIDS and lipodystrophy: implications for clinical management in resource-limited settings. J Int AIDS Soc 2015;18:19033.

11. Cofrancesco J Jr, Freedland E, McComsey G. AIDS Patient Care STDS 2009;23:5-18.

12. Benedini S, Terruzzi I, Lazzarin A, Luzi L. Recombinant human growth hormone: rationale for use in the treatment of HIV-associated lipodystrophy. Bio Drugs 2008;22:101-12.

13. Tripathy SK, Agrawala RK, Baliarsinha AK. Endocrine alterations in HIV-infected patients. Indian J Endocrinol Metab 2015;19:143-7.

14. Quintas RC, de França ER, de Petribú KC, Ximenes RA, Quintas LF, Cavalcanti EL, et al. Treatment of facial lipoatrophy with polymethylmethacrylate among patients with human immunodeficiency virus/ acquired immunodeficiency syndrome (HIV/AIDS): impact on the quality of life. Int J Dermatol 2014;53:497-502.

15. Carr A, Samaras K, Burton S, Law M, Freund J, Chisholm DJ, et al. AIDS 1998; 12: F51-8.

16. Masiá M, Padilla S, García N, Jarrin I, Bernal E, López N, et al.Endothelial function is impaired in HIV-infected patients with lipodystrophy. Antivir Ther 2010;15:101-10.

17. Valantin MA, Aubron-Olivier C, Ghosn J, Laglenne E, Pauchard M, Schoen $\mathrm{H}$, et al. Polylactic acid implants (New-Fill) to correct facial lipoatrophy in HIV-infected patients: results of the open-label study VEGA. AIDS 2003;17:2471-7.

18. Singhania R, Kotler DP. Lipodystrophy in HIV patients: its challenges and management approaches. HIV AIDS (Auckl) 2011;3:135-43.

19. Kharitonenkov A, DiMarchi R. FGF21 Revolutions: Recent Advances Illuminating FGF21 Biology and Medicinal Properties. Trends Endocrinol Metab 2015; 26:608-17.
20. Kim KH, Lee MS.FGF21 as a mediator of adaptive responses to stress and metabolic benefits of anti-diabetic drugs. J Endocrinol 2015;226:R1-16.

21. Spolcová A, Holubová M, Mikulášková B, Nagelová V, Stofková A, Lacinová Z, et al. Changes in FGF21 Serum Concentrations and Liver mRNA Expression in an Experimental Model of Complete Lipodystrophy and Insulin-Resistant Diabetes. Physiol Res 2014 4;63:483-90.

22. Gaich G, Chien JY, Fu H, Glass LC, Deeg MA, Holland WL, et al. The effects of LY2405319, an FGF21 analog, in obese human subjects with type 2 diabetes. Cell Metab 2013;18: 333-40.

23. Dong JQ, Rossulek M, Somayaji VR, Baltrukonis D, Liang Y, Hudson $\mathrm{K}$, et al. Pharmacokinetics and pharmacodynamicsof PF-05231023, a novel long-acting FGF21 mimetic, in a first-in-human study. Br J Clin Pharmacol 2015;80:1051-29.

24. Kajimura S, Saito M. A new era in brown adipose tissue biology: molecular control of brown fat development and energy homeostasis. Annu Rev Physiol 2014; 76: 225-49.

25. Fitch KV, Anderson EJ, Hubbard JL, Carpenter SJ, Waddell WR, Caliendo AM, et al. Effects of a lifestyle modification program in HIV-infected patients with the metabolic syndrome. AIDS 2006; 20:1843-50.

26. Benedini S, Perseghin G, Terruzzi I, Scifo P, Invernizzi PL, Del Maschio A, et al. Effect of L-acetylcarnitine on body composition in HIV-related lipodystrophy. Horm Metab Res 2009;41:840-5.

27. Sheth SH, Larson RJ. The efficacy and safety of insulin-sensitizing drugs in HIV-associated lipodystrophy syndrome: a meta-analysis of randomized trials. BMC Infect Dis 2010;10:183.

28. Lindegaard B, Hvid T, Grøndahl T, Frosig C, Gerstoft J, Hojman P, et al. Expression of Fibroblast Growth Factor-21 in Muscle Is Associated with Lipodystrophy, Insulin Resistance and Lipid Disturbances in Patients with HIV. PLoS One 2013;8:e55632.

29. Domingo P, Gallego-Escuredo JM, Domingo JC, Gutiérrez Mdel M, Mateo MG, Fernández I, et al. Serum FGF21 levels are elevated in association with lipodystrophy, insulin resistance and biomarkers of liver injury in HIV-1-infected patients. AIDS 2010;24:2629-37.

30. Zhang X, Yeung DC, Karpisek M, Stejskal D, Zhou ZG, Liu F, et al. Serum FGF21 levels are increased in obesity and are independently associated with the metabolic syndrome in humans. Diabetes 2008; 57:1246-53.

31. Muise ES, Azzolina B, Kuo DW, El-Sherbeini M, Tan Y, Yuan X, et al. Adipose fibroblast growth factor 21 is up-regulated by peroxisome proliferator-activated receptor gamma and altered metabolic states. Mol Pharmacol 2008; 74: 403-12.

32. Kharitonenkov A, Shiyanova TL, Koester A, Ford AM, Micanovic R, Galbreath EJ, et al. FGF-21 as a novel metabolic regulator. J Clin Invest 2005;115: 1627-35.

33. Bobbert T, Schwarz F, Fischer-Rosinsky A, Pfeiffer AF, Mohlig M, Mai K. et al. Fibroblast growth factor 21 predicts the metabolic syndrome and type 2 diabetes in Caucasians. Diabetes Care 2013; 36:145e149.

34. Itoh N. FGF21 as a Hepatokine, Adipokine, and Myokine in Metabolism and Diseases. Front Endocrinol (Lausanne) 2014;5:107.

How to cite this article: Benedini S, Luzi L. Lipodystrophy HIV-related and FGF21: A new marker to follow the progression of lipodystrophy? J Transl Intern Med 2016; 4: 150-54. 